\title{
Smoothening of $\mathrm{Cu}$ films grown on $\mathrm{Si}(001)$
}

\author{
R. A. Lukaszew, ${ }^{\text {a) }}$ Y. Sheng, C. Uher, and R. Clarke \\ Randall Laboratory of Physics, University of Michigan, Ann Arbor, Michigan 48109-1120
}

(Received 2 November 1999; accepted for publication 13 December 1999)

\begin{abstract}
We report an in situ study of the molecular-beam epitaxy growth and annealing of $\mathrm{Cu}(001)$ films grown on hydrogen-terminated $\mathrm{Si}(001)$ substrates, resulting in a promising approach to achieve smooth epitaxial morphology. Using correlated reflection high-energy electron diffraction and scanning tunneling microscopy data, we find a temperature interval below the onset of silicide formation where a dramatic smoothening of the epitaxial $\mathrm{Cu}$ surfaces occurs. Our measurements indicate that a reduction in roughness is possible in this regime because the annealing is controlled by lateral diffusion kinetics. (C) 2000 American Institute of Physics. [S0003-6951(00)04106-1]
\end{abstract}

One of the outstanding issues in metal epitaxy is the role of surface diffusion kinetics and how this controls the morphology of the film under low-temperature-growth conditions. ${ }^{1}$ In particular, the appearance of surface roughness as a result of noise ${ }^{2}$ or step-edge barriers (ErlichSchwöbel effect ${ }^{3}$ ) is an important challenge to the development of applications of epitaxial metal layers. In the case of heteroepitaxy, strain can also play an important role in roughening. ${ }^{4,5}$

In this letter, we report results of an in situ study of the mass transport kinetics of $\mathrm{Cu}$ grown on hydrogen-terminated $\mathrm{Si}(100)$. We show that there exists a relatively narrow temperature window within which a smooth epitaxial $\mathrm{Cu}$ layer morphology can be achieved. We find that the activation energy for lateral (terrace) diffusion is significant $\left[E_{d}\right.$ $=0.36 \pm 0.03 \mathrm{eV}$ (Ref. 6) $]$ and can dominate in the lowtemperature range explored in this study. However, even though the lateral mass transport is limited in this regime we show that a mild annealing process yields relatively large increases in the terrace size and a concomitant smoothing of the surface morphology.

Annealing the sample to achieve smoother surfaces is effective if the annealing temperature $T$ is below the roughening transition temperature $T_{R}$ of the surface. ${ }^{7}$ For $T$ $>T_{R}$, the surface is rough and annealing does not lead to smoothening, but $T$ should be high enough to promote sufficiently fast dynamics. However, for copper grown on silicon it has been reported ${ }^{8}$ that a silicide is formed at the interface with an onset of $473 \mathrm{~K}$. This, therefore, imposes an upper limit to the annealing temperature for this system.

Our studies were performed in a VG $80 \mathrm{M}$ molecularbeam epitaxy (MBE) chamber equipped with an in situ ultrahigh-vacuum (UHV) scanning tunneling microscope [(STM) RHK model STM100] and a real-time reflection high-energy electron diffraction (RHEED) imaging system. ${ }^{9}$ The films were grown under UHV conditions on $\mathrm{Si}(001)$ substrates cleaned ultrasonically with acetone and alcohol for $15 \mathrm{~min}$ each and etched with HF (10\% by volume) for $30 \mathrm{~s}$. The substrates were held at ambient temperature during the growth. The copper was evaporated from a Knudsen cell at a rate of $0.3 \AA / s$. The thickness of the films was $\sim 2000 \AA$.

a)Electronic mail: lukaszew@umich.edu
Two films were annealed by heating from room temperature up to 420 and $473 \mathrm{~K}$, respectively, at a rate of $1 \mathrm{~K} / \mathrm{min}$. Subsequent films were annealed isothermally at different temperatures in the range $395 \mathrm{~K} \leqslant T<445 \mathrm{~K}$ for $2-4 \mathrm{~h}$. The RHEED pattern was monitored continuously during the growth and annealing. The various stages of growth and annealing were also evaluated by STM imaging of the film surface.

After the growth of $\sim 2000 \AA$ of $\mathrm{Cu}$ the RHEED pattern had a symmetrical spotty appearance characteristic of threedimensional growth [inset (a) in Fig. 1]. ${ }^{10}$ One of the films was annealed by increasing the temperature at a rate of 1 $\mathrm{K} / \mathrm{min}$ from ambient to $473 \mathrm{~K}$ where the copper RHEED pattern faded. The second film was annealed at the same rate but only up to $420 \mathrm{~K}$ where the heating process showed its effect through the sharpening of the copper streaks, indicating that the crystallinity has improved significantly as a result of the annealing [inset (b) in Fig. 1]. A plot of 1/FWHM (full width at half maximum) of the specular RHEED streak versus temperature, shows two distinct regimes: a gradual narrowing for $T<395 \mathrm{~K}$, and a more rapid evolution for $T>395 \mathrm{~K}$ (Fig. 1). We have also observed that for $T>445 \mathrm{~K}$, the RHEED pattern becomes very diffuse, indicating that the surface becomes rough again marking the on-

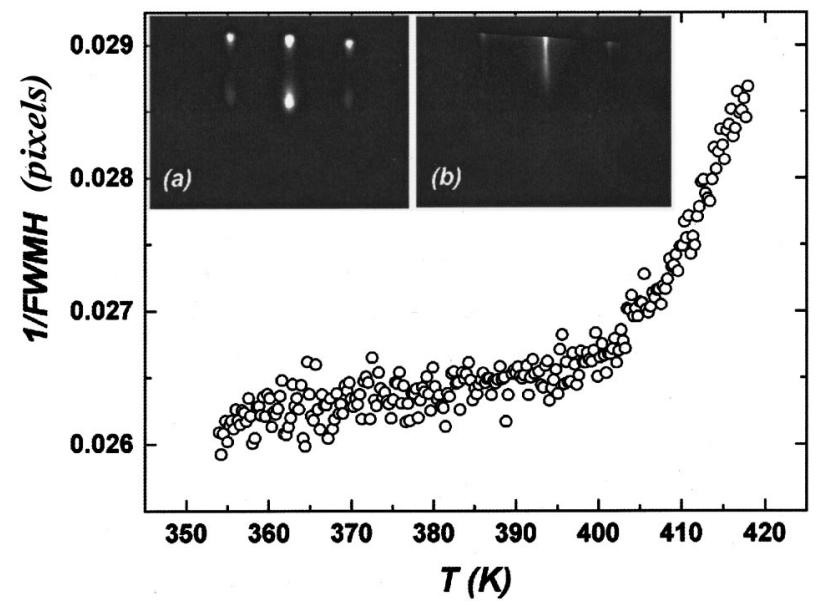

FIG. 1. Annealing of a $2000 \AA \mathrm{Cu}$ film. Inverse width of the RHEED specular streak as a function of temperature. Inset: (100) zone RHEED images of the film, (a) as grown and (b) after annealing at $423 \mathrm{~K}$ for $4 \mathrm{~h}$. The $\mathrm{Cu}$ axes are rotated in plane by $45^{\circ}$ relative to silicon. 

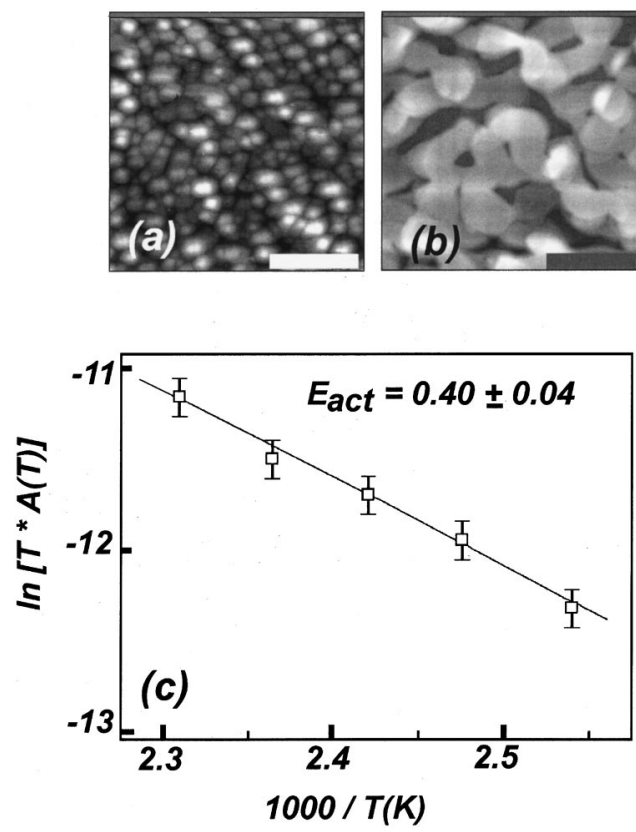

FIG. 2. STM images of a 2000-Å-thick Cu film: (a) as grown; (b) after annealing at $423 \mathrm{~K}$ for $4 \mathrm{~h}$ (scanned area: $0.3 \mu \mathrm{m} \times 0.3 \mu \mathrm{m}$, scale bar $=100 \mathrm{~nm}$ ); and (c) Arrhenius plot of $T^{*} A(T)$ vs $1 / T$, where $A(T)$ $\propto(1 / T) \exp \left[-\left(E_{\text {act }}\right) / k_{B} T\right]$. The $A(T)$ values were obtained from linear fits to Eq. (1) for various temperature values in the range $393 \mathrm{~K}<T<433 \mathrm{~K}$.

set of silicide formation. At approximately $470 \mathrm{~K}$ the copper RHEED pattern disappears. Subsequent films were annealed isothermally in the fast regime $(T \geqslant 395 \mathrm{~K}$, but below the silicide onset temperature $T<445 \mathrm{~K}$ ). Figure 2(a) shows the STM image after growth at room temperature. The rms roughness of the unannealed surface is $\sim 70 \AA$ and the grain size is small $(\sim 160 \AA)$ due to interfacial strain (the lattice mismatch for an in-plane rotation of $45^{\circ}$ between the $\mathrm{Si}$ and $\mathrm{Cu}$ lattices is $\sim 6 \%$ ) coupled to kinetic parameters that lead to deviations from ideal epitaxial growth. Figure 2(b), on the other hand, shows the STM image after annealing the film at $420 \mathrm{~K}$ for $4 \mathrm{~h}$. The surface of the film now has large islands (average size $800 \AA$ ) and the mean rms roughness has decreased to $\sim 10 \AA$.

It is worth mentioning here that the temperature $T_{t}(\sim 395 \mathrm{~K})$ measured for the onset of the fast diffusion process corresponds to $(0.28 \pm 0.01) T_{M}$, where $T_{M}$ is the melting point of $\mathrm{Cu}$ (in $\mathrm{K}$ ). We find a similar relationship when we use the value of $T_{t}(\sim 600 \mathrm{~K})$ determined during the annealing of fcc $\mathrm{Rh}(111)$ films MBE grown on mica. ${ }^{11}$

In order to analyze the smoothing kinetics of this system, we followed a similar analysis to that performed by Zuo and Wendelken ${ }^{12}$ in their study of homoepitaxial $\mathrm{Cu}(001)$. In the temperature range $333 \mathrm{~K}<T<368 \mathrm{~K}$ (i.e., below the onset of rapid annealing that we have identified), they found that the average terrace width $L(t)$ follows a modified $t^{1 / 3}$ law, consistent with kinetics driven by the line tension of curved step edges ${ }^{13,14}$ with an activation energy of $1.10 \mathrm{eV}$ :

$$
L^{3}(t)-L^{3}\left(t_{0}\right)=A(T)\left(t-t_{0}\right),
$$

where $A(T) \propto(1 / T) \exp \left[-\left(E_{\text {act }}\right) / k_{B} T\right]$, is a temperaturedependent factor. ${ }^{12}$ Our data agree with a $t^{1 / 3}$ law behavior below the onset of fast diffusion. In addition, for the temperature range $395 \mathrm{~K} \leqslant T<445 \mathrm{~K}$, our experimental values of the exponents for the time variable $\left[\left(t-t_{0}\right)\right.$ in Eq. (1) $]$ are in

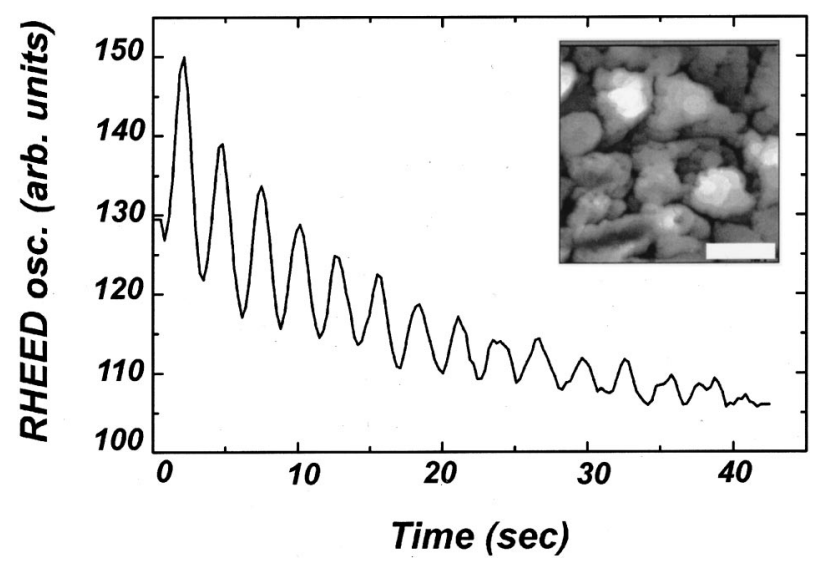

FIG. 3. RHEED oscillations observed after further copper deposition. Inset: STM image after disappearance of RHEED oscillations (scanned area: $0.3 \mu \mathrm{m} \times 0.3 \mu \mathrm{m}$, scale bar=100 nm).

the range $0.98 \pm 0.02$, consistent with the scaling in Eq. (1). Afterwards, from the least-squares fit to the data, the prefactor $A(T)$ was obtained for the $395 \mathrm{~K} \leqslant T<445 \mathrm{~K}$ temperature interval. From an Arrhenius plot of $\ln [T A(T)]$ vs $1 / T$ [Fig. 2 (c)] we obtain an activation energy of $0.40 \pm 0.04 \mathrm{eV}$.

Thus, we see that there is a narrow temperature interval below the onset of silicide formation $(395 \mathrm{~K} \leqslant T<445 \mathrm{~K}$ ) where the activation energy is considerably smaller than that measured earlier $(1.1 \mathrm{eV})$ at lower temperatures. ${ }^{12}$ The value we find $\left(E_{\text {act }}=0.40 \pm 0.04 \mathrm{eV}\right)$ is very close to reported activation energies for surface diffusion $\left[E_{d}=0.36 \pm 0.03 \mathrm{eV}\right.$ (Ref. 6)], indicating that the fast smoothing of the surface is dominated by lateral diffusion in the temperature interval considered. Therefore, it is possible to mitigate the effects of limited mass transport by an annealing process in which modest heating of the as-grown film leads to an overall smoothening of the surface. The mechanism responsible for the observed transition in the mass transport kinetics at the surface is not known at this time. It is possible that the hydrogen termination at the $\mathrm{Si}-\mathrm{Cu}$ interface plays a role in the observed behavior. ${ }^{15}$ Further studies of $\mathrm{Cu}$ films grown and annealed on a hydrogen-free $(2 \times 1)$ reconstructed $\mathrm{Si}(001)$ surface may clarify this point.

Provided the annealing is performed at relatively low temperatures $(T<473 \mathrm{~K})$, we found that this process yields flat surfaces which have the crystallographic form of copper, not the silicide. Subsequent copper growth at room temperature on a film annealed at $T=423 \mathrm{~K}$ for $4 \mathrm{~h}$ exhibited RHEED oscillations. Many oscillations were observed for all the films grown, fading progressively as growth proceeds [Fig. 3(a)]. The presence of step-edge barriers favors the growth of smaller islands on top of the larger ones leading to mounding, and thus the disappearance of the RHEED oscillations ${ }^{16}$ as the growth continues [Fig. 3(b)].

In order to further characterize the nature of the annealed $\mathrm{Cu} / \mathrm{Si}$ heteroepitaxial surface we performed a series of experiments on the scaling behavior of the growth. These measurements parallel earlier experiments on homoepitaxial $\mathrm{Cu}$ growth (irreversible growth by Ernst et al. ${ }^{17}$ and reversible growth by Zuo and Wendelken), ${ }^{18}$ and we have extended these studies into the thick film regime where the growth exponent $\alpha$ can be measured. Scaling analysis ${ }^{19}$ was applied to the STM images obtained after further copper was depos- 
ited on the annealed surface. The rms roughness (interface width) $\xi$ for length $L$ and average thickness $\langle h\rangle$, for a system containing $N$ sites with single valued heights $h_{i}$, is defined by the expression

$$
\xi(L,\langle h\rangle)=\left[N^{-1} \sum_{i}^{N}\left[h_{i}-\langle h\rangle\right]^{2}\right]^{1 / 2} .
$$

Theory ${ }^{19}$ predicts that when $\langle h\rangle \rightarrow 0, \xi$ scales as

$$
\xi(\langle h\rangle) \propto\langle h\rangle^{\beta},
$$

where $\beta$ is the dynamic roughness exponent, and when $\langle h\rangle$ $\rightarrow \infty$ it scales as

$$
\xi(L) \propto L^{\alpha},
$$

where $\alpha$ is the static roughness exponent. The exponent $\beta$ was determined from a series of STM images obtained after the deposition of 3-15 ML of $\mathrm{Cu}$ deposited on the smooth surface obtained after annealing. The exponent $\alpha$ on the other hand, was obtained from a single image obtained after $2000 \AA$ of further $\mathrm{Cu}$ deposition. Accordingly, $\alpha$ was derived from Eq. (4) where $L$ corresponds to a segment of the STM scan measured along a direction parallel to the surface. The plot $\log \xi$ vs $\log L$ exhibited a linear region for values of $L$ smaller than the grain size $\left(d_{s}\right)$ and after that it saturated. The slope of this straight line was found to be $\alpha=1.03$ \pm 0.03 . On the other hand, the exponent $\beta$ was evaluated from Eq. (3). From a plot of $\log \xi$ vs $\log \langle h\rangle$, a straight line is obtained with a slope $\beta=0.25 \pm 0.01$. Another measure of scaling is the length scale associated with coarsening. As $\langle h\rangle$ increases, the average grain size $d_{s}$ increases according to

$$
d_{s} \propto\langle h\rangle^{\beta / \alpha} .
$$

From the plot $\log d_{s}$ vs $\log \langle h\rangle$ a value for $\beta / \alpha=0.25 \pm 0.01$ is obtained, and considering the value obtained previously for $\alpha(\cong 1)$, we estimate a value of 0.25 for $\beta$, consistent with the value derived from Eq. (3).

Our values agree with those reported by Zuo and Wendelken ${ }^{18}$ for the reversible homoepitaxial growth of $\mathrm{Cu}(001)$ at room temperature and also with theoretical models. ${ }^{20}$ It is worth noting here that when the substrate was held at lower temperature $(T=260 \mathrm{~K})$, which inhibits lateral diffusion, additional copper deposition yielded a "chevron" bifurcation of the RHEED pattern shortly after the RHEED oscillations faded, characteristic of faceting. The angle of the chevron was measured to be $\sim 15^{\circ}$. This observation is in agreement with the Ernst et al. ${ }^{17}$ findings regarding "pyramid-like" structures where the sides of the pyramids corresponded to the (115) vicinal surface in homoepitaxial irreversible (001) copper growth at $200 \mathrm{~K}$.
In summary, we have found a temperature interval where activated surface diffusion is the dominant mass transport mechanism for annealing copper epitaxially grown on hydrogen-terminated (001) silicon before the onset of the silicide formation. This process enhances the surface flatness of the films. Annealing a $2000 \AA$ copper film grown at room temperature on hydrogen-terminated (100) Si at $423 \mathrm{~K}$ for 4 $\mathrm{h}$ yields a surface with rms roughness of $10 \AA$. Our in situ RHEED and STM imaging yielded complementary information regarding the surface quality of the films.

The authors wish to acknowledge fruitful conversations with Professor Jacques Amar (University of Toledo) and Professor Ratna Naik (Wayne State University).

\footnotetext{
${ }^{1}$ H. Brune, Surf. Sci. Rep. 31, 121 (1998).

${ }^{2}$ F. Family and T. Vicsek, J. Phys. A 18, L75 (1985).

${ }^{3}$ G. Erlich and F. Hudda, J. Chem. Phys. 44, 1039 (1966); R. L. Schwöbel, J. Appl. Phys. 40, 614 (1969); R. L. Schwöbel and E. J. Shipsey, ibid. 37, 3682 (1966); J. Villain, J. Phys. I 1, 19 (1992).

${ }^{4}$ D. J. Eaglesham and M. Cerullo, Phys. Rev. Lett. 64, 1943 (1990); Y.-W. Mo, D. E. Savage, B. S. Swartzentruber, and M. G. Lagally, ibid. 65, 1020 (1990).

${ }^{5}$ E. Hahn, E. Kampshoff, N. Wälchli, and K. Kern, Phys. Rev. Lett. 74, 1803 (1995)

${ }^{6}$ H. Dürr, J. F. Wendelken, and J.-K. Zuo, Surf. Sci. 328, L527 (1995).

${ }^{7}$ W. K. Burton and N. Cabrera, Discuss. Faraday Soc. 5, 33 (1949); J. D. Weeks, in Ordering in Strongly Fluctuating Condensed Matter Systems, edited by T. Riste (Plenum, New York, 1980); H. Van Beijen and I. Nolden, in Structures and Dynamics of Surfaces III, edited by W. Schommers and P. von Blanckenhagen (Springer, Berlin, 1987).

${ }^{8}$ R. Padyath, J. Seth, S. V. Babu, and L. J. Matienzo, J. Appl. Phys. 75, 2326 (1993).

${ }^{9}$ D. Barlett, C. W. Snyder, B. G. Orr, and R. Clarke, Rev. Sci. Instrum. 62, 1263 (1991); data acquisition using KSA400, k-Space Assoc. Inc., Ann Arbor, MI 48109.

${ }^{10}$ B. G. Demczyk, R. Naik, G. Auner, C. Kota, and U. Rao, J. Appl. Phys. 75, 1956 (1994)

${ }^{11}$ F. Tsui, J. Wellman, C. Uher, and R. Clarke, in Evolution of Epitaxial Structure and Morphology, edited by A. Zangwill, D. Jesson, D. Chambliss, and R. Clarke (MRS, Pittsburgh, PA 1996).

${ }^{12}$ J.-K. Zuo and J. F. Wendelken, Phys. Rev. Lett. 70, 1662 (1993).

${ }^{13}$ J. Villain, Europhys. Lett. 2, 531 (1986).

${ }^{14}$ A. Rettori and J. Villain, J. Phys. (France) 49, 257 (1988).

${ }^{15}$ There have been some reports in the literature that hydrogen dramatically speeds up the kinetics at $\mathrm{Cu}$ surfaces; K. Haug, Z. Zhang, D. John, C. F. Walters, D. M. Zehner, and W. E. Plummer, Phys. Rev. B 55, R10233 (1997).

${ }^{16}$ J. Tersoff, A. W. D. van der Fon, and R. M. Tromp, Phys. Rev. Lett. 72, 266 (1994).

${ }^{17}$ H.-J. Ernst, F. Fabre, R. Folkerts, and J. Lapujoulade, Phys. Rev. Lett. 72, 112 (1994).

${ }^{18}$ J.-K. Zuo and J. F. Wendelken, Phys. Rev. Lett. 78, 2791 (1997).

${ }^{19}$ F. Family, Physica A 168A, 561 (1990).

${ }^{20}$ M. Siegert and M. Plishcke, Phys. Rev. Lett. 73, 1517 (1994); J. Amar Phys. Rev. B 60, R11317 (1999).
} 